\title{
Dexamethasone Suppresses Oxysterol-Induced Differentiation of Monocytic Cells
}

\author{
Yonghae Son, ${ }^{1}$ Bo-Young Kim, ${ }^{1}$ Seong-Kug Eo, ${ }^{2}$ Young Chul Park, ${ }^{3}$ and Koanhoi Kim ${ }^{1}$ \\ ${ }^{1}$ Department of Pharmacology, Pusan National University School of Medicine, Yangsan, Gyeongnam 50612, Republic of Korea \\ ${ }^{2}$ College of Veterinary Medicine and Bio-Safety Research Institute, Chonbuk National University, Iksan, \\ Jeonbuk 54596, Republic of Korea \\ ${ }^{3}$ Department of Microbiology \& Immunology, Pusan National University School of Medicine, Yangsan, \\ Gyeongnam 50612, Republic of Korea \\ Correspondence should be addressed to Koanhoi Kim; koanhoi@pusan.ac.kr
}

Received 18 February 2016; Revised 14 May 2016; Accepted 15 May 2016

Academic Editor: Giuseppe Filomeni

Copyright (C) 2016 Yonghae Son et al. This is an open access article distributed under the Creative Commons Attribution License, which permits unrestricted use, distribution, and reproduction in any medium, provided the original work is properly cited.

Oxysterol like 27-hydroxycholesterol (27OHChol) has been reported to induce differentiation of monocytic cells into a mature dendritic cell phenotype. We examined whether dexamethasone (Dx) affects $27 \mathrm{OHChol}$-induced differentiation using THP-1 cells. Treatment of monocytic cells with Dx resulted in almost complete inhibition of transcription and surface expression of CD80, CD83, and CD88 induced by $27 \mathrm{OHChol}$. Elevated surface levels of MHC class I and II molecules induced by $27 \mathrm{OHChol} \mathrm{were}$ reduced to basal levels by treatment with Dx. A decreased endocytosis ability caused by $27 \mathrm{OHChol} \mathrm{was} \mathrm{recovered} \mathrm{by} \mathrm{Dx.} \mathrm{We}$ also examined effects of Dx on expression of CD molecules involved in atherosclerosis. Increased levels of surface protein and transcription of CD105, CD137, and CD166 by treatment with $27 \mathrm{OHChol} \mathrm{were} \mathrm{significantly} \mathrm{inhibited} \mathrm{by} \mathrm{cotreatment} \mathrm{with} \mathrm{Dx.}$ These results indicate that Dx inhibits 27OHChol-induced differentiation of monocytic cells into a mature dendritic cell phenotype and expression of $\mathrm{CD}$ molecules whose levels are associated with atherosclerosis. In addition, we examined phosphorylation of AKT induced by $27 \mathrm{OHChol} \mathrm{and} \mathrm{effect} \mathrm{of} \mathrm{Dx,} \mathrm{where} \mathrm{cotreatment} \mathrm{with} \mathrm{Dx} \mathrm{inhibited} \mathrm{the} \mathrm{phosphorylation} \mathrm{of} \mathrm{AKT.} \mathrm{The} \mathrm{current} \mathrm{study}$ reports that Dx regulates oxysterol-mediated dendritic cell differentiation of monocytic cells.

\section{Introduction}

Dexamethasone (Dx), a glucocorticoid, is widely used in treatment of chronic inflammatory and immunological diseases because it is a potent immune-suppressive and antiinflammatory drug $[1,2]$. Dx inhibits secretion of inflammatory mediators [3] and Th1 type immune response [4]. Dx also downregulates DC functions in vivo and in vitro [5]. Dx alters function of monocyte-derived dendritic cells (MoDCs) from cord blood [6] and inhibits maturation of dendritic cells (DCs) by redirecting differentiation of a subset of cells [7]. In addition, Dx inhibits the antigen presentation of DCs [8]. These reports indicate that Dx affects immunological responses by regulating differentiation and function of DCs.

DCs are potent antigen-presenting cells in the immune system and are critically involved in the initiation of primary immune responses and autoimmune responses that occur in diseases including atherosclerosis $[9,10]$. DCs can be generated in vitro using monocytes. Human blood CD14 ${ }^{+}$ monocytes differentiate into DCs when cultured in combination with granulocyte-macrophage colony stimulating factor (GM-CSF) and interleukin-4 (IL-4), and addition of tumor necrosis factor- $\alpha$ (TNF- $\alpha$ ) induces maturation into CD $83^{\text {high }}$-DC $[10,11]$. Lipopolysaccharide (LPS), TNF- $\alpha$, and calcium ionophore rapidly induce the differentiation of blood monocytes into DC-like cells [12]. THP-1, a human monocytic cell line, also differentiates into DCs. Treatment of THP-1 cells with cytokines and ionomycin results in differentiation into mature DCs (mDCs) [13]. 27-Hydroxycholesterol (27OHChol) also rapidly induces the differentiation of THP1 cells into mDCs [14] with high expression of $\mathrm{mDC}$-specific markers such as CD80, CD83, CD86, and CD88. However, there is no information regarding drugs that regulate 27OHChol-mediated differentiation into mDCs. 
We were interested in the question whether Dx can affect DCs differentiation induced by $27 \mathrm{OHChol}$. In the current study, we determine effects of Dx on expression of $\mathrm{mDC}$ markers and MHC molecules induced by $27 \mathrm{OHChol,}$ using THP-1 cells. In addition, we also examined whether Dx modulated expression of CD molecules, such as CD105, CD137, and CD166, which are associated with atherosclerosis.

\section{Materials and Methods}

2.1. Cell Culture and Reagents. THP-1 cells purchased from American Type Culture Collection (ATCC, Manassas, VA 20108) were maintained with RPMI 1640 containing 10\% fetal bovine serum (FBS) in the presence of penicillin and streptomycin. 27OHChol was purchased from Research Plus, Inc. (Bayonne, NJ, USA). Dx was purchased from ENZO Life Science, Inc. (Farmingdale, NY). Fluorescein isothiocyanate(FITC-) conjugated dextran $(40 \mathrm{kDa})$ was purchased from Sigma-Aldrich (St. Louis, MO, USA). Primary antibodies were purchased from Santa Cruz Biotechnology (Santa Cruz, CA, USA). Alexa Fluor 488-conjugated secondary antibodies for FACS analysis were purchased from Invitrogen (Eugene, Oregon).

2.2. Dextran-FITC Uptake Assay. After incubation with Dx, 27OHChol, and PMA, THP-1 cells were resuspended in culture medium containing $1 \mathrm{mg} / \mathrm{mL}$ of FITC-conjugated dextran and incubated for $30 \mathrm{~min}$ at $37^{\circ} \mathrm{C}$ or $4^{\circ} \mathrm{C}$ (for background control). Cells were washed with cold phosphate buffered saline (PBS) containing 1\% FBS, and flow cytometry was performed for analysis of uptake of FITC-dextran.

\subsection{Reverse Transcriptase-Polymerase Chain Reaction (RT-} $P C R)$. RT-PCR was performed as previously described [14]. In brief, total RNAs were reverse-transcribed for $1 \mathrm{~h}$ at $42^{\circ} \mathrm{C}$ with Moloney Murine Leukemia Virus reverse transcriptase, followed by PCR analysis. Glyceraldehyde-3-phosphate dehydrogenase (GAPDH) was amplified as a control with primers of $5^{\prime}$-GAGTCAACGGATTTGGTCGT (forward) and $5^{\prime}$ TGTGGTCATGAGTCCTTCCA (reverse). Primer pairs of $\mathrm{CD}$ molecules were designed using free online primer design tool primer 3. PCR products were visualized using ethidium bromide after electrophoresis on agarose gels.

2.4. Quantitative Real-Time Polymerase Chain Reaction. Quantitative real-time PCR was performed as previously described [15]. In brief, quantitative real-time PCR was performed in triplicate in 96-well plates containing SYBR Green PCR Master Mix and $10 \mathrm{pM}$ forward primer and reverse primer for CD molecules and glyceraldehyde-3-phosphate dehydrogenase (GAPDH). The sequence of CD molecule primers was forward 5' -TGGTGCTGGCTGGTCTTTC and reverse $5^{\prime}$-CTGTGCCACTTCTTTCACTTCC (CD80); forward $5^{\prime}$-TCCTGAGCTGCGCCTACAG and reverse $5^{\prime}$-GCAGGGCAAGTCCACATCTT (CD83); forward 5'-GTGGTCCGGGAGGAGTACTTT and reverse $5^{\prime}$-GCCGTTTGTCGTGGCTGTA (CD88); forward $5^{\prime}$-CATCCTTGAAGTCCATGTCCTCTT and reverse $5^{\prime}$-GCCAGGTGCCATTTTGCTT (CD105); forward 5' -TCACTGCCTGGGGGCAGGAT and reverse $5^{\prime}$ GGCGGGGTCACAGAGGATGC (CD137); forward $5^{\prime}$-TCCTGCCGTCTGCTCTTCT and reverse $5^{\prime}$-TTCTGAGGTACGTCAAGTCGG (CD166). Primers for GAPDH were forward 5' -ATGGGGAAGGTGAAGGTCG and reverse 5' -GGGGTCAT TGATGGCAACAATA.

2.5. Flow Cytometric Analysis. After incubation with Dx and 27OHChol, THP-1 cells were harvested and incubated for $2 \mathrm{~h}$ at $4^{\circ} \mathrm{C}$ with antibodies against CD80, CD83, CD88, CD105, CD137, CD166, and major histocompatibility complex (MHC) class I and II molecules, followed by washing and incubation with fluorescent dye-conjugated secondary antibodies. Cells were washed and resuspended in 1\% paraformaldehyde in PBS. Flow cytometry was performed for analysis of fluorescence.

2.6. ELISA. After incubation for $48 \mathrm{~h}$ with $\mathrm{Dx}$ and $27 \mathrm{OHChol}$, the cells were analyzed using ELISA kit for phospho-AKT (R\&D Systems, Inc. Minneapolis, MD, USA), following the manufacturer's instructions.

2.7. Statistical Analysis. Statistical analyses were performed using one-way ANOVA, followed by Tukey's multiple comparison tests, using GraphPad PRISM (version 5.0).

\section{Results}

3.1. Attenuated Expression of mDC Markers by Treatment with $D x$. We determined expression of $\mathrm{mDC}$ markers to examine whether Dx affects differentiation of monocytic cells induced by $27 \mathrm{OHChol}$. Treatment with $27 \mathrm{OHChol} \mathrm{resulted}$ in significantly increased transcription of $\mathrm{mDC}$ markers CD80, CD83, and CD88, and addition of Dx resulted in their attenuated transcription, as determined by real-time PCR (Figure 1(a)). The levels of CD80, CD83, and CD88 were increased 4.03-, 4-, and 4.37-fold by treatment with $27 \mathrm{OHChol}$, respectively, compared with unstimulated control, and the increases were reduced to be 1.74-, 1.86-, and 1.1-fold, respectively, by cotreatment with $0.1 \mu \mathrm{M}$ of Dx and almost completely inhibited to basal levels by treatment with $1 \mu \mathrm{M}$ and $10 \mu \mathrm{M}$ of Dx.

We also examined effects of Dx on surface expression of mDC markers (Figure 1(b)). In agreement with results of realtime PCR, flow cytometric analyses showed increases of CD molecules by treatment with $27 \mathrm{OHChol}$. The percentages of control cells positive for CD80, CD83, and CD88 were $8.9 \%$, $2.2 \%$, and $2.1 \%$, respectively, which increased to $59.8 \%, 54.6 \%$, and $34.7 \%$, respectively, by treatment with $27 \mathrm{OHChol}$. However, the increases of $\mathrm{mDC}$ markers induced by $27 \mathrm{OHChol}$ were almost completely inhibited to control by treatment with Dx. Collectively, these results indicate that Dx reduced not only transcription but also surface expression of $\mathrm{mDC}$ markers in monocytic cells.

3.2. Attenuated Expression of MHC Molecules by Treatment with $D x$. Flow cytometry was performed to examine whether Dx influenced expression of MHC class molecules (Figure 2). The percentage of control cells positive for MHC class I 


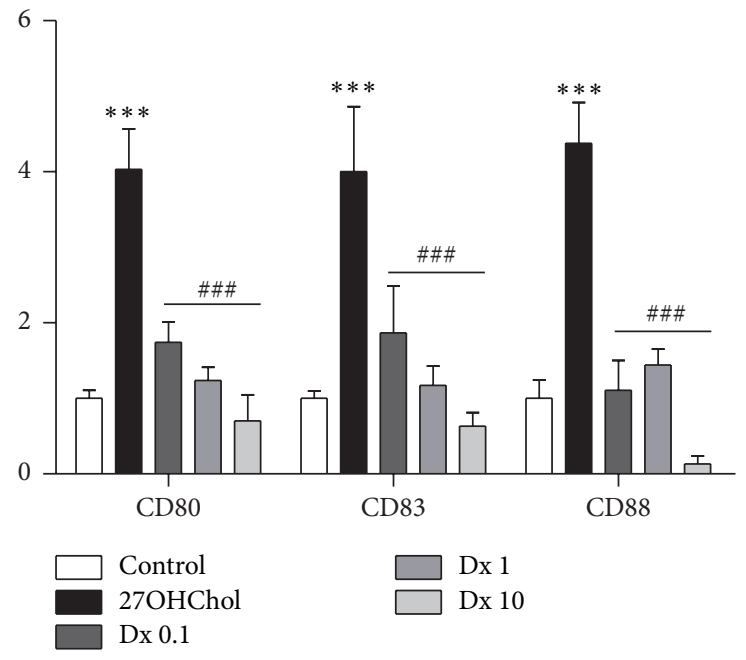

(a)
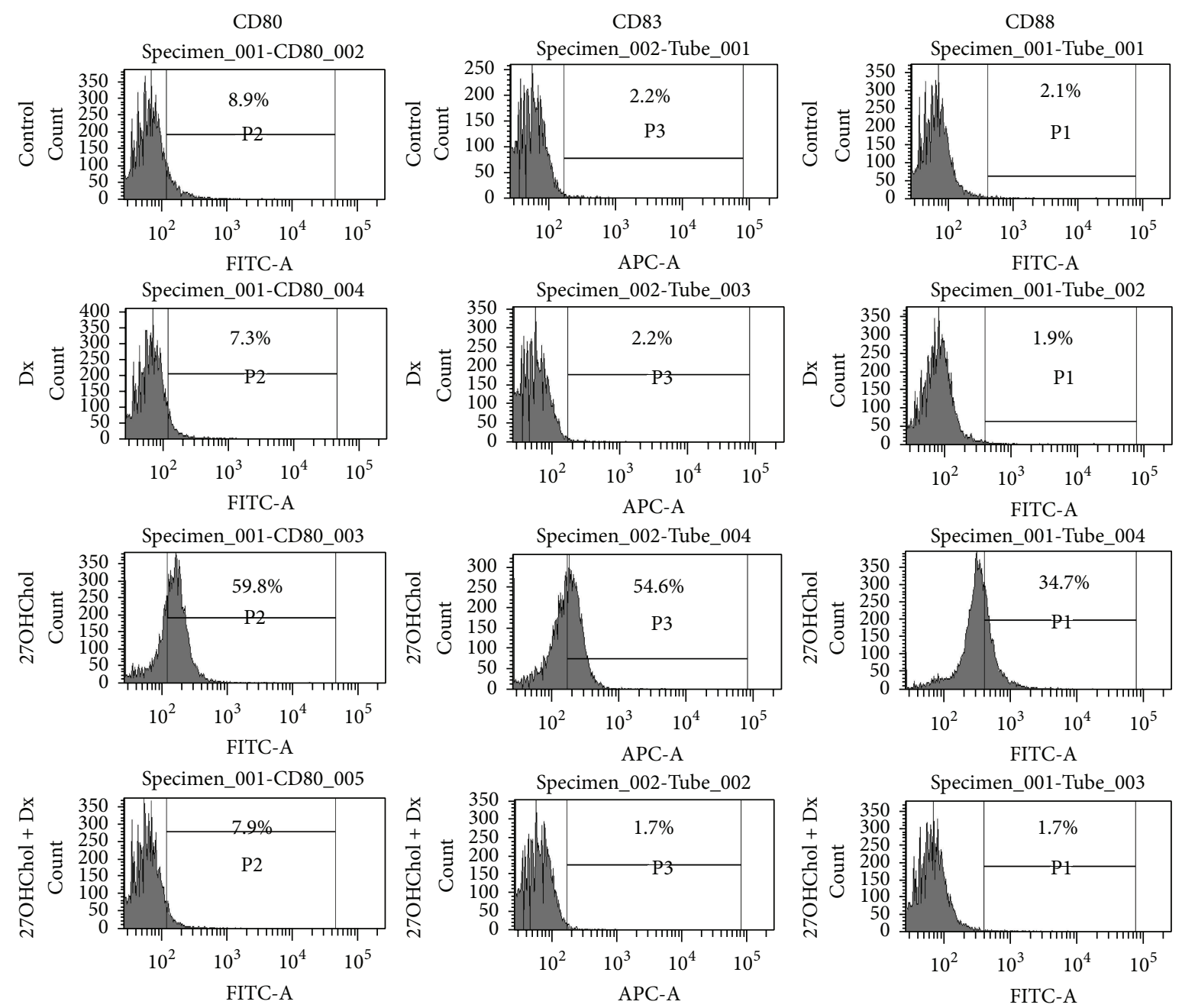

(b)

FIGURE 1: Effects of Dx on the transcription and surface expression of mDC markers induced by $27 \mathrm{OHChol}$. (a) THP- 1 cells $\left(1 \times 10^{6}\right.$ cells $/ 60 \mathrm{~mm}$ culture dish) were cultured for $48 \mathrm{~h}$ with $2.5 \mu \mathrm{g} / \mathrm{mL}$ of $27 \mathrm{OHChol} \mathrm{with} \mathrm{or} \mathrm{without} 0.1,1$, or $10 \mu \mathrm{M}$ of Dx. Transcription of CD80, CD83, and CD88 was analyzed by real-time PCR. Data are expressed as mean $\pm \mathrm{SD}\left(n=3\right.$ replicates/group). ${ }^{* * *} P<0.001$ versus control; ${ }^{\# \# \#} P<0.001$ versus $27 \mathrm{OHChol}$. (b) THP-1 cells $\left(1 \times 10^{6}\right.$ cells $/ 60 \mathrm{~mm}$ culture dish) were cultured for $48 \mathrm{~h}$ in the presence of $2.5 \mu \mathrm{g} / \mathrm{mL}$ of $27 \mathrm{OHChol} \mathrm{with} \mathrm{or} \mathrm{without} 1 \mu \mathrm{M}$ of Dx. Cells were immunostained with antibodies against CD80, CD83, and CD88 and analyzed by flow cytometry. Results are representative of three independent experiments. 

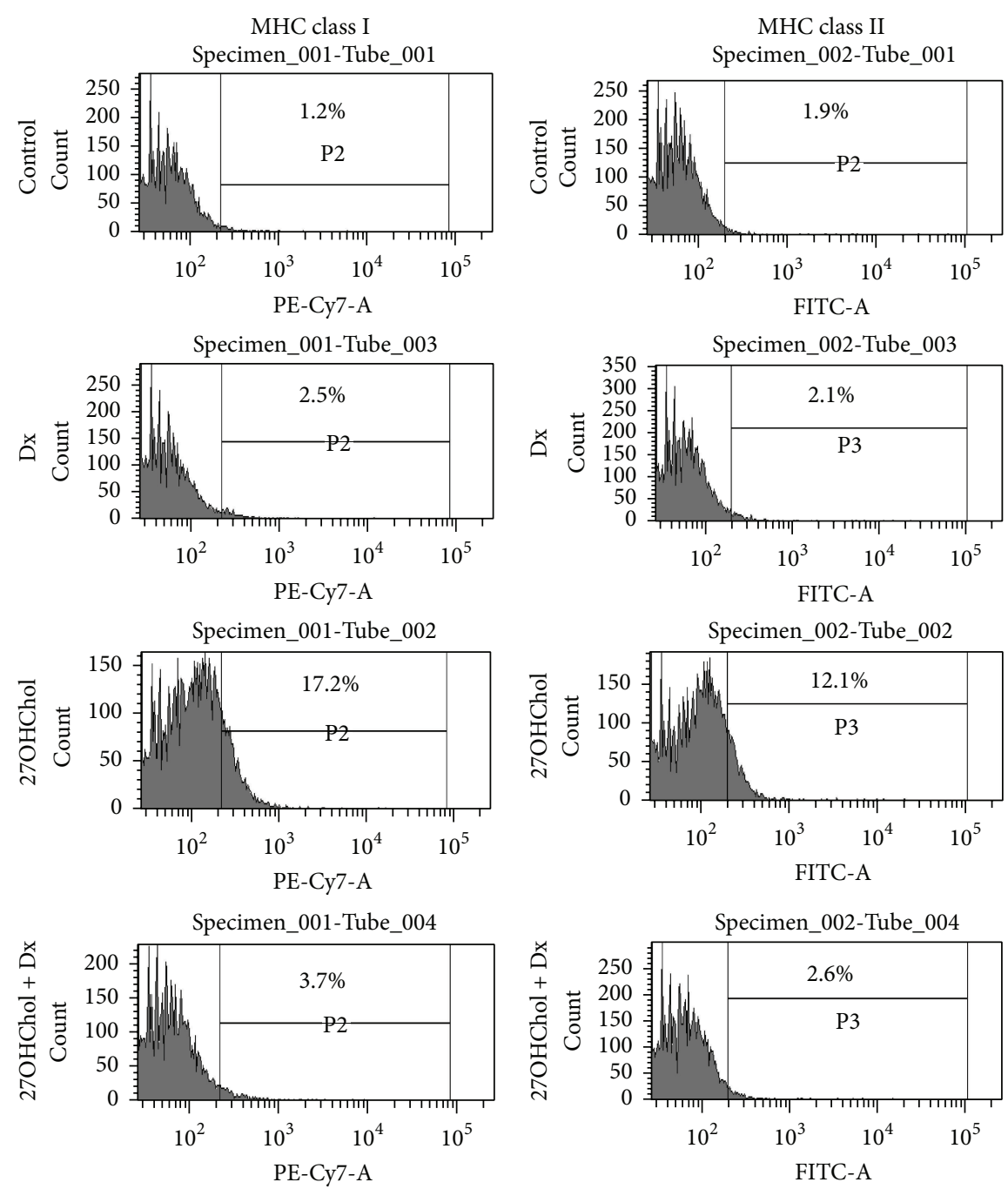

FIGURE 2: Effects of Dx on expression of MHC class I and II molecules induced by $27 \mathrm{OHChol}$. After incubation for $48 \mathrm{~h}$ with $27 \mathrm{OHChol}$ $(2.5 \mu \mathrm{g} / \mathrm{mL})$ with or without Dx $(1 \mu \mathrm{M})$, the stimulated THP- 1 cells $\left(1 \times 10^{6}\right.$ cells $/ 60 \mathrm{~mm}$ culture dish $)$ were immunostained for MHC classes I and II. Fluorescence was analyzed by flow cytometry. Results are representative of three independent experiments.

molecule was $1.2 \%$, which showed a significant increase to $17.2 \%$ by treatment with $27 \mathrm{OHChol}$. Similarly, the percentage of control cells positive for MHC class II molecule was $1.9 \%$, which was increased to $12.1 \%$ by treatment with $27 \mathrm{OHChol}$. However, the increased levels of MHC class molecules were reduced to control level by cotreatment with Dx. These results indicate that $\mathrm{Dx}$ inhibited surface expression of $\mathrm{MHC}$ molecules.

3.3. Recovered Endocytic Function by Treatment with Dx. We performed an endocytosis ability test to determine whether $\mathrm{Dx}$ affected functional alteration induced by $27 \mathrm{OHChol}$ on monocytic cells (Figure 3 ). The percentage of control cells exhibiting endocytic activity was $19.5 \%$, which was significantly reduced to $9.8 \%$ by treatment with $27 \mathrm{OHChol}$ and increased to $15.2 \%$ by cotreatment with Dx. Treatment with phorbol 12-myristate 13 -acetate (PMA) resulted in an increase in percentage of cells exhibiting endocytic activity up to $40.4 \%$, which was decreased to $23.7 \%$ by cotreatment with
Dx. However, there was no change in endocytic function by treatment with Dx alone. These results indicate that endocytic function of monocytic cells modified by treatment with 27OHChol or PMA was recovered by Dx.

3.4. Attenuated Expression of Atherosclerosis-Associated CD Molecules by Treatment with Dx. We examined whether Dx influenced expression of CD molecules CD105, CD137, and CD166, which are associated with atherosclerosis. Transcription of CD105, CD137, and CD166 was elevated, and addition of Dx resulted in decreased transcription of the genes (Figure 4(a)). Compared with control, the levels of CD105, CD137, and CD166 were increased 6.8-, 5.7-, and 4.9-fold, respectively, by treatment with $27 \mathrm{OHChol,} \mathrm{and} \mathrm{the}$ increases were reduced to basal levels by cotreatment with $1 \mu \mathrm{M}$ and $10 \mu \mathrm{M}$ of $\mathrm{Dx}$. We also examined effects of $\mathrm{Dx}$ on surface expression of the molecules by flow cytometry (Figure 4(b)). The percentage of control cells positive for CD105 was $3.2 \%$, which was increased to $16.5 \%$ by treatment 

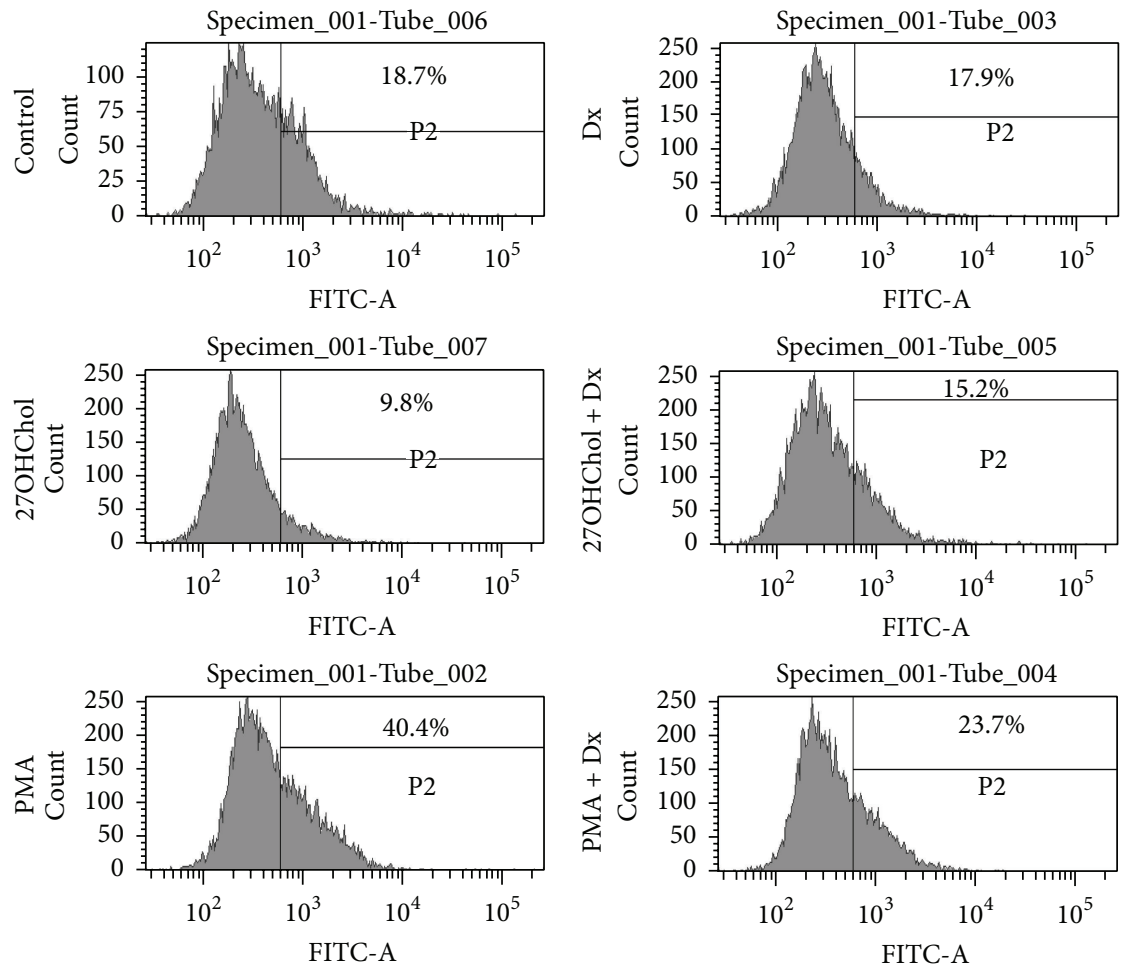

FIgURE 3: Effects of Dx on functional alteration of monocytic cells induced by $27 \mathrm{OHChol}$. After incubation for $48 \mathrm{~h}$ with $27 \mathrm{OHChol}$ $(2.5 \mu \mathrm{g} / \mathrm{mL})$ with or without Dx $(1 \mu \mathrm{M})$ and PMA $(200 \mathrm{nM})$, THP-1 cells were treated with $1 \mathrm{mg} / \mathrm{mL}$ of FITC-conjugated dextran for $1 \mathrm{~h}$. The cells were analyzed by flow cytometry. Results are representative of three independent experiments.

with $27 \mathrm{OHChol}$ and was reduced to $5.1 \%$ by cotreatment with Dx. The percentage of CD137-positive control cells was 7.6\%, which was increased to $16.4 \%$ by treatment with $27 \mathrm{OHChol,}$ which was reduced to $7.3 \%$ by Dx. The percentage of CD166positive control cells was $4.7 \%$, which was increased to $14.7 \%$ by treatment with $27 \mathrm{OHChol}$ and was reduced to $5.1 \%$ by Dx. These results indicate that expression of CD105, CD137, and CD166 induced by $27 \mathrm{OHChol} \mathrm{was} \mathrm{significantly} \mathrm{inhibited} \mathrm{by}$ cotreatment with Dx at the levels of transcription and protein.

\subsection{Attenuated Phosphorylation of AKT by Treatment with} $D x$. To determine the 27OHChol-mediated pathway and the effects of Dx, we examined using ELISA kit for p-AKT (Figure 5). Phosphorylation of AKT was increased to be 8.7fold by stimulation with $27 \mathrm{OHChol}$, which was reduced to be 4.2-, 4.1-, and 4.4-fold, respectively, by treatment with 0.1 , 1 , and $10 \mu \mathrm{M}$ of Dx. This result showed that Dx affected the phosphorylation of AKT increased by $27 \mathrm{OHChol}$.

\section{Discussion}

CD molecules overexpressed by $27 \mathrm{OHChol}$ not only are $\mathrm{mDC}$-specific markers but also are involved in activation of T- and B-lymphocytes. CD80, also known as B7-1 or CD28 ligand, is a surface molecule involved in activation of Blymphocytes [16]. CD83, a coactivator of T-lymphocytes, is required in development of $\mathrm{CD} 4{ }^{+} \mathrm{T}$ cells [17]. CD88, known as $\mathrm{C} 5 \mathrm{a}$ receptor, plays key roles in secretion of IL-12 and in Th2 type immune response [18]. Therefore, downregulated expression of CD molecules indicates that Dx will negatively regulate, in addition to differentiation of monocytic cells, activation of T-/B-lymphocytes. This idea is in line with previous studies reporting that $\mathrm{Dx}$ inhibited activation of T-/B-lymphocytes via enhanced apoptosis [19, 20]. These studies showed direct effects of Dx against activation of $\mathrm{T}$ and $\mathrm{B}$ cells whereas our result showed an indirect effect of Dx on activation of the cells. Activated T- and B-lymphocytes can promote atherosclerosis [21, 22]. It is possible that downregulated expression of CD80, CD83, and CD88 by Dx can affect pathogenesis of atherosclerosis. Further studies are needed in order to assess correlation between downregulated expression of CD molecules and atherosclerosis using an animal model treated with Dx.

MHC molecules play a role in antigen presentation captured by endo- or phagocytosis on the cell surface and, in contrast to monocytes or immature DCs (imDCs), are highly expressed on mDCs [9]. The expressed MHC molecules stimulate naïve $\mathrm{T}$ cells in secondary lymph nodes and induce adaptive immunity. Experimental evidence indicates that Dx can modulate expression of MHC molecules, thereby affecting antigen presentation. Dx downregulated expression of MHC class II on human monocytic cells stimulated with IFN- $\gamma$ [23] and also inhibited differentiation into DCs and antigen presentation of DCs by MHC class II pathway induced by cytokines like GM-CSF and IL-4 [8]. The current study added new information to the previous reports that Dx reduces levels of surface MHC class I and II molecules induced by $27 \mathrm{OHChol}$. The reduced expression of MHC I 


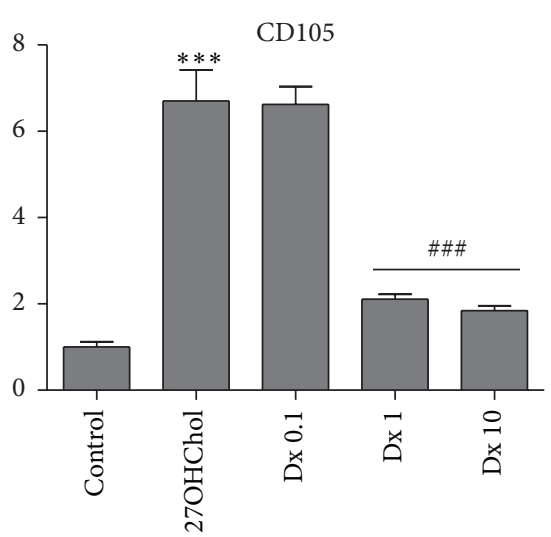

CD105
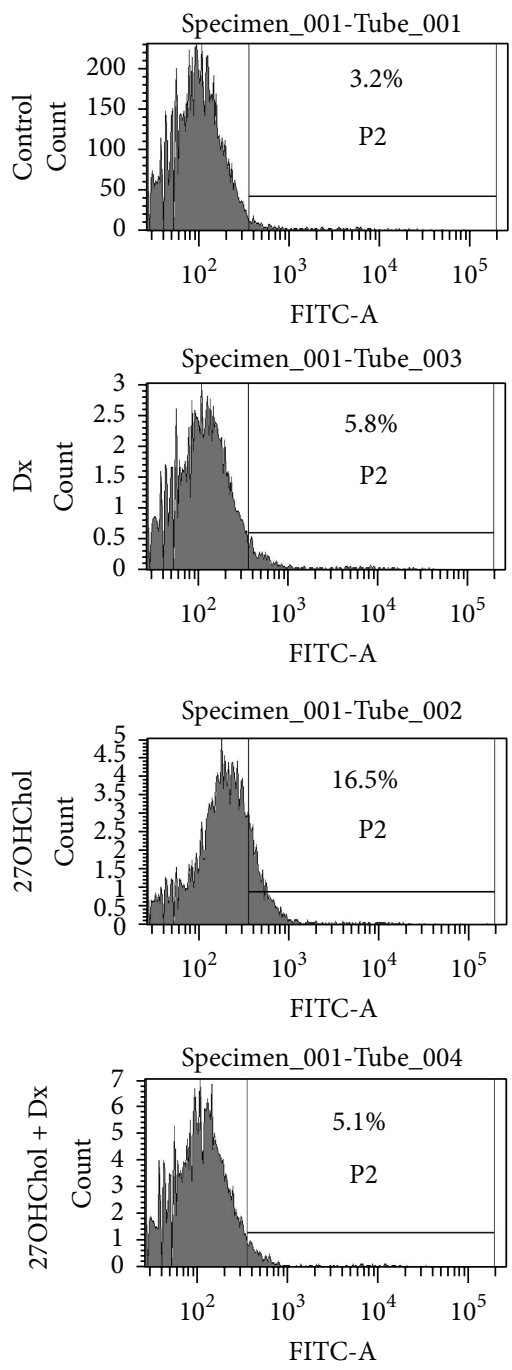

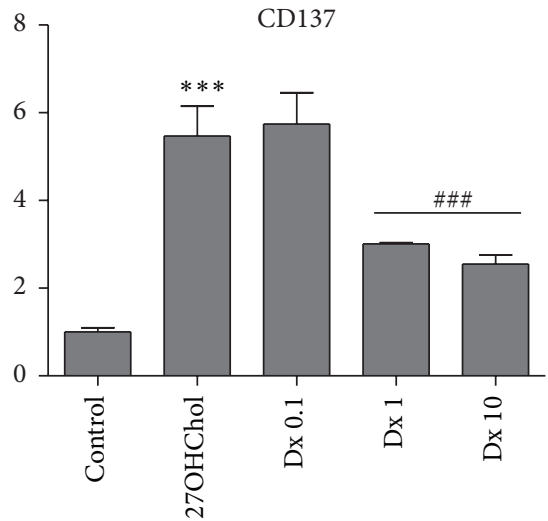

(a)
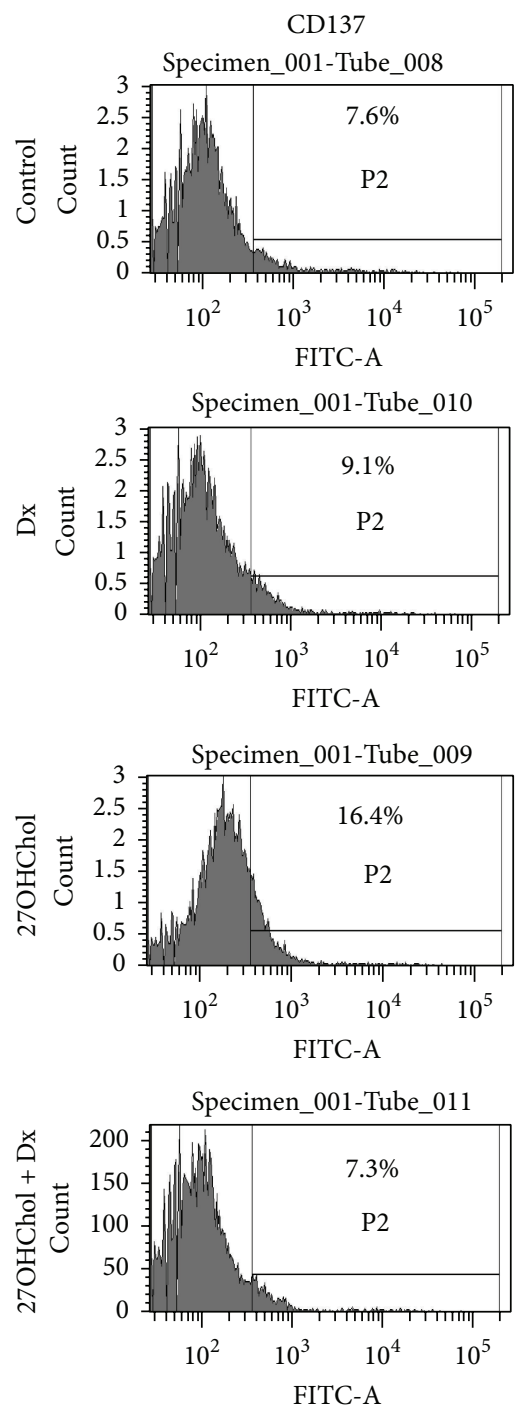

(b)

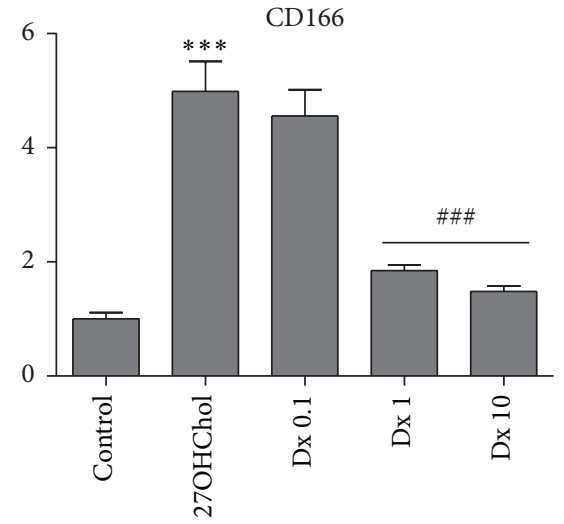

CD166
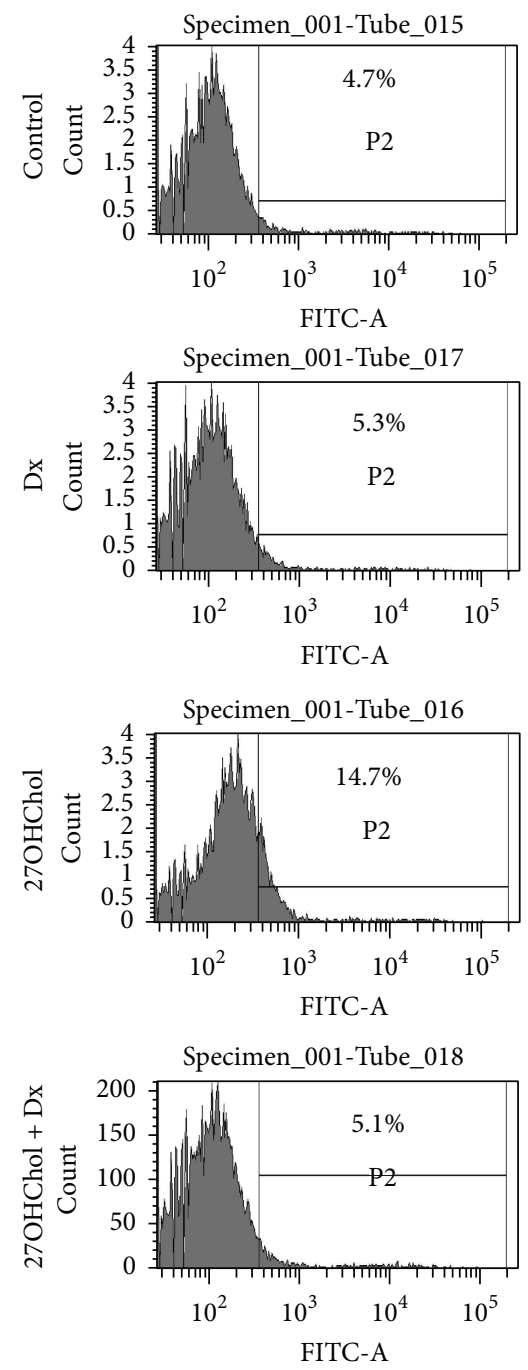

FIGURE 4: Effects of Dx on expression of atherosclerosis-associated CD molecules induced by 27OHChol. (a) After incubation with 27OHChol $(2.5 \mu \mathrm{g} / \mathrm{mL})$ for $48 \mathrm{~h}$ with or without $0.1,1$, or $10 \mu \mathrm{M}$ of Dx, transcription of CD105, CD137, and CD166 was analyzed by real-time PCR. Data are expressed as mean \pm SD $\left(n=3\right.$ replicates/group). ${ }^{* * *} P<0.001$ versus control; ${ }^{\# \# \# ~} P<0.001$ versus $27 \mathrm{OHChol}$. (b) THP-1 cells $\left(1 \times 10^{6}\right.$ cells $/ 60 \mathrm{~mm}$ culture dish) were cultured for $48 \mathrm{~h}$ with $2.5 \mu \mathrm{g} / \mathrm{mL}$ of $27 \mathrm{OHChol}$ with or without $1 \mu \mathrm{M}$ of Dx. The harvested cells were immunostained with antibodies against CD105, CD137, and CD166 and analyzed by flow cytometry. Results are representative of three independent experiments. 


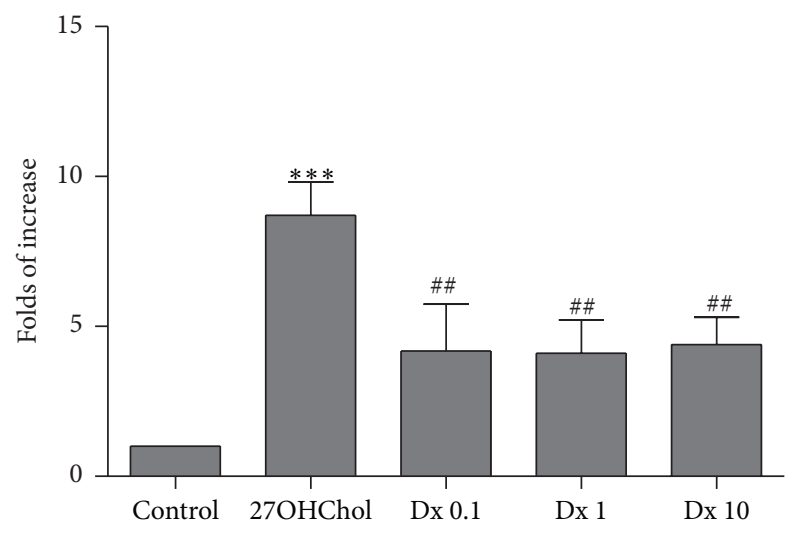

FIGURE 5: Effect of Dx on phosphorylation of AKT induced by 27OHChol. After incubation with $27 \mathrm{OHChol}(2.5 \mu \mathrm{g} / \mathrm{mL})$ for $48 \mathrm{~h}$ with or without $0.1,1$, or $10 \mu \mathrm{M}$ of Dx, lysates from the cells were analyzed with ELISA kit for p-AKT. Data are expressed as mean \pm $\mathrm{SD}\left(n=3\right.$ replicates/group). ${ }^{* * *} P<0.001$ versus control; ${ }^{\# \#} P<0.05$ versus $27 \mathrm{OHChol.}$

and II suggests that Dx can indirectly modulate activation of $\mathrm{T}$ cells through downregulation of MHC class molecules on 27OHChol-stimulated monocytic cells.

DCs capture antigens through endo- or phagocytosis. The captured antigens are digested in endo- or phagosome and presented by MHC molecules on cell surface. DCs show a different endocytosis activity in each stage $[9,24]$. Antigen-uptake activity is high in immature DCs (imDCs) state but very low in $\mathrm{mDC}$ state. Dx-mediated recovery in endocytic functional change of monocytic cells induced by $27 \mathrm{OHChol} \mathrm{suggests} \mathrm{that} \mathrm{Dx} \mathrm{can} \mathrm{modulate} \mathrm{antigen} \mathrm{uptake} \mathrm{and}$ presentation of it on cell surface because antigen presentation is influenced by endocytosis activity. We consider that Dxmediated recovery of endocytosis activity is associated with inhibition of $27 \mathrm{OHChol-mediated} \mathrm{differentiation.} \mathrm{This} \mathrm{idea} \mathrm{is}$ in agreement with the study by Piemonti et al., which showed that Dx recovered the endocytosis activity of MoDCs induced by GM-CSF and IL-4 through inhibition of differentiation from imDCs to mDCs [25]. Taken together, these findings indicate that Dx recovered changes of endocytic activity in monocytic cells differentiated by cytokines and oxysterol.

CD137 (known as 4-1BB), which is highly expressed in human atherosclerosis, promotes development of plaque inflammation [26], and its deficiency results in reduced atherosclerosis induced by hypercholesterolemia [27]. CD105 is overexpressed in atherosclerotic tissues [28, 29], and CD166 is a marker of atherosclerosis (USA patent number US8603829 B2). Therefore, the finding of upregulated expression of $\mathrm{CD}$ molecules by treatment with $27 \mathrm{OHChol}$, which agrees with the previous study [14], indicates that $27 \mathrm{OHChol}$ is likely to increase their expression in atherosclerosis, thereby causing deterioration of the disease. In addition, decreased expression of the proatherogenic CD molecules will have beneficial effects by treatment with Dx because expression of CD molecules is correlated with severity of atherosclerosis. We think that the results of the current study provide a mechanism through which Dx induces suppressed atherosclerosis in an animal model of atherosclerosis [30].

We determined signaling pathways stimulated by $27 \mathrm{OHChol}$. Some studies reported that $27 \mathrm{OHChol} \mathrm{promoted}$ inflammations and immune response through estrogen receptor- (ER-) $\alpha[28,31]$, and TLR4/NF- $\kappa$ B signaling pathway was involved in the oxysterol-mediated responses [32]. We had studied that $27 \mathrm{OHChol} \mathrm{induced} \mathrm{the} \mathrm{upregulation} \mathrm{of} \mathrm{sol-}$ uble CD14 (sCD14) and MMP-9 expression, AKT pathway was involved in the upregulation on monocytic cells [33]. However, which signaling pathways involved in the differentiation of monocytic cells induced by $27 \mathrm{OHChol} \mathrm{had} \mathrm{not} \mathrm{been}$ reported. Our ELISA result suggests that $27 \mathrm{OHChol} \mathrm{increases}$ phosphorylation of AKT, and Dx inhibited the increase. This data means that the 27OHChol-mediated differentiation mediated through AKT pathways. About signaling pathways involved in the differentiation, we perform more study.

Results of the current study suggest that Dx can modify immune responses by inhibiting differentiation of monocytic cells and expression of MHC molecules induced by $27 \mathrm{OHChol}$ and by reversing endocytic function. We propose that Dx disturbs immune response in a milieu rich in oxidatively modified cholesterol molecules like atherosclerotic plaques.

\section{Competing Interests}

The authors declare that they have no competing interests.

\section{Acknowledgments}

This research was supported by the Basic Science Research Program through the National Research Foundation of Korea (NRF) funded by the Ministry of Education (NRF2014R1A1A4A01006878).

\section{References}

[1] D. T. Boumpas, "Glucocorticoid therapy for immune-mediated diseases: basic and clinical correlates," Annals of Internal Medicine, vol. 119, no. 12, pp. 1198-1208, 1993.

[2] J.-B. Wasserfallen and J. N. Baraniuk, "Clinical use of inhaled corticosteroids in asthma," Journal of Allergy and Clinical Immunology, vol. 97, no. 1, pp. 177-182, 1996.

[3] A. Nehmé and J. Edelman, "Dexamethasone inhibits high glucose-, TNF- $\alpha$ - and IL- $1 \beta$-induced secretion of inflammatory and angiogenic mediators from retinal microvascular pericytes, Investigative Ophthalmology and Visual Science, vol. 49, no. 5, pp. 2030-2038, 2008.

[4] A. Franchimont, J. Galon, M. Gadina et al., "Inhibition of Th1 immune response by glucocorticoids: dexamethasone selectively inhibits IL-12-induced Stat4 phosphorylation in T lymphocytes," Journal of Immunology, vol. 164, no. 4, pp. 1768-1774, 2000.

[5] M. Moser, T. De Smedt, T. Sornasse et al., "Glucocorticoids down-regulate dendritic cell function in vitro and in vivo," European Journal of Immunology, vol. 25, no. 10, pp. 2818-2824, 1995.

[6] E. S. Mainali, T. Kikuchi, and J. G. Tew, "Dexamethasone inhibits maturation and alters function of monocyte-derived 
dendritic cells from cord blood," Pediatric Research, vol. 58, no. 1, pp. 125-131, 2005.

[7] R. Matasić, A. B. Dietz, and S. Vuk-Pavlović, "Dexamethasone inhibits dendritic cell maturation by redirecting differentiation of a subset of cells," Journal of Leukocyte Biology, vol. 66, no. 6, pp. 909-914, 1999.

[8] J. Pan, D. Ju, Q. Wang et al., "Dexamethasone inhibits the antigen presentation of dendritic cells in MHC class II pathway," Immunology Letters, vol. 76, no. 3, pp. 153-161, 2001.

[9] R. M. Steinman, "The dendritic cell system and its role in immunogenicity," Annual Review of Immunology, vol. 9, no. 1, pp. 271-296, 1991.

[10] L.-J. Zhou and T. F. Tedder, "CD14+ blood monocytes can differentiate into functionally mature CD83+ dendritic cells," Proceedings of the National Academy of Sciences of the United States of America, vol. 93, no. 6, pp. 2588-2592, 1996.

[11] F. Sallusto and A. Lanzavecchia, "Efficient presentation of soluble antigen by cultured human dendritic cells is maintained by granulocyte/macrophage colony-stimulating factor plus interleukin 4 and downregulated by tumor necrosis factor $\alpha$," Journal of Experimental Medicine, vol. 179, no. 4, pp. 11091118, 1994.

[12] L. A. Lyakh, G. K. Koski, W. Telford, R. E. Gress, P. A. Cohen, and N. R. Rice, "Bacterial lipopolysaccharide, TNF- $\alpha$, and calcium ionophore under serum-free conditions promote rapid dendritic cell-like differentiation in CD14+ monocytes through distinct pathways that activate NF- $\kappa \mathrm{B}$," Journal of Immunology, vol. 165, no. 7, pp. 3647-3655, 2000.

[13] C. Berges, C. Naujokat, S. Tinapp et al., "A cell line model for the differentiation of human dendritic cells," Biochemical and Biophysical Research Communications, vol. 333, no. 3, pp. 896907, 2005.

[14] Y. Son, S.-M. Kim, S.-A. Lee, S.-K. Eo, and K. Kim, “Oxysterols induce transition of monocytic cells to phenotypically mature dendritic cell-like cells," Biochemical and Biophysical Research Communications, vol. 438, no. 1, pp. 161-168, 2013.

[15] S.-H. Kang, J.-H. Lee, K.-H. Choi, B.-Y. Rhim, and K. Kim, "Roles of ERK and NF- $\kappa$ B in interleukin-8 expression in response to heat shock protein 22 in vascular smooth muscle cells," Korean Journal of Physiology and Pharmacology, vol. 12, no. 4, pp. 171-176, 2008.

[16] F. E. Katz, M. Parkar, K. Stanley, L. J. Murray, E. A. Clark, and M. F. Greaves, "Chromosome mapping of cell membrane antigens expressed on activated B cells," European Journal of Immunology, vol. 15, no. 1, pp. 103-106, 1985.

[17] Y. Fujimoto, L. Tu, A. S. Miller et al., "CD83 expression influences $\mathrm{CD}^{+} \mathrm{T}$ cell development in the thymus," Cell, vol. 108, no. 6, pp. 755-767, 2002.

[18] S. H. Gavett, D. J. O’Hearn, X. Li, S.-K. Huang, F. D. Finkelman, and M. Wills-Karp, "Interleukin 12 inhibits antigen-induced airway hyperresponsiveness, inflammation, and Th2 cytokine expression in mice," The Journal of Experimental Medicine, vol. 182, no. 5, pp. 1527-1536, 1995.

[19] K. Andréau, C. Lemaire, V. Souvannavong, and A. Adam, "Induction of apoptosis by dexamethasone in the B cell lineage," Immunopharmacology, vol. 40, no. 1, pp. 67-76, 1998.

[20] K. Xing, B. Gu, P. Zhang, and X. Wu, "Dexamethasone enhances programmed cell death 1 (PD-1) expression during T cell activation: an insight into the optimum application of glucocorticoids in anti-cancer therapy," BMC Immunology, vol. 16, article 39, 2015.
[21] H. M. Perry and C. A. McNamara, "Refining the role of B cells in atherosclerosis," Arteriosclerosis, Thrombosis, and Vascular Biology, vol. 32, no. 7, pp. 1548-1549, 2012.

[22] E. Profumo, B. Buttari, L. Saso, R. Capoano, B. Salvati, and R. Riganò, "T lymphocyte autoreactivity in inflammatory mechanisms regulating atherosclerosis," The Scientific World Journal, vol. 2012, Article ID 157534, 9 pages, 2012.

[23] L. M. Schwiebert, R. P. Schleimer, S. F. Radka, and S. J. Ono, "Modulation of MHC class II expression in human cells by dexamethasone," Cellular Immunology, vol. 165, no. 1, pp. 12-19, 1995.

[24] J. Banchereau, F. Briere, C. Caux et al., "Immunobiology of dendritic cells," Annual Review of Immunology, vol. 18, pp. 767$811,2000$.

[25] L. Piemonti, P. Monti, P. Allavena et al., "Glucocorticoids affect human dendritic cell differentiation and maturation," The Journal of Immunology, vol. 162, no. 11, pp. 6473-6481, 1999.

[26] P. S. Olofsson, L. Å. Söderström, D. Wågsäter et al., "CD137 is expressed in human atherosclerosis and promotes development of plaque inflammation in hypercholesterolemic mice," Circulation, vol. 117, no. 10, pp. 1292-1301, 2008.

[27] S.-M. Kim, B.-Y. Kim, S.-A. Lee et al., "27-Hydroxycholesterol and 7alpha-hydroxycholesterol trigger a sequence of events leading to migration of CCR5-expressing Th1 lymphocytes," Toxicology and Applied Pharmacology, vol. 274, no. 3, pp. 462470, 2014.

[28] C. D. DuSell, M. Umetani, P. W. Shaul, D. J. Mangelsdorf, and D. P. McDonnell, "27-Hydroxycholesterol is an endogenous selective estrogen receptor modulator," Molecular Endocrinology, vol. 22, no. 1, pp. 65-77, 2008.

[29] M. Piao and O. Tokunaga, "Significant expression of endoglin (CD105), TGF $\beta-1$ and TGF $\beta$ R-2 in the atherosclerotic aorta: an immunohistological study," Journal of Atherosclerosis and Thrombosis, vol. 13, no. 2, pp. 82-89, 2006.

[30] K. Asai, C. Funaki, T. Hayashi et al., "Dexamethasone-induced suppression of aortic atherosclerosis in cholesterol-fed rabbits. Possible mechanisms," Arteriosclerosis, Thrombosis, and Vascular Biology, vol. 13, no. 6, pp. 892-899, 1993.

[31] M. Umetani, H. Domoto, A. K. Gormley et al., “27Hydroxycholesterol is an endogenous SERM that inhibits the cardiovascular effects of estrogen," Nature Medicine, vol. 13, no. 10, pp. 1185-1192, 2007.

[32] S. Gargiulo, P. Gamba, G. Testa et al., "Relation between TLR4/ NF- $\kappa \mathrm{B}$ signaling pathway activation by 27 -hydroxycholesterol and 4-hydroxynonenal, and atherosclerotic plaque instability," Aging Cell, vol. 14, pp. 569-581, 2015.

[33] S.-M. Kim, S.-A. Lee, B.-Y. Kim, S.-S. Bae, S.-K. Eo, and K. Kim, "27-Hydroxycholesterol induces recruitment of monocytic cells by enhancing CCL2 production," Biochemical and Biophysical Research Communications, vol. 442, no. 3-4, pp. 159-164, 2013. 


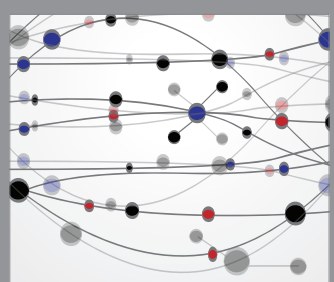

The Scientific World Journal
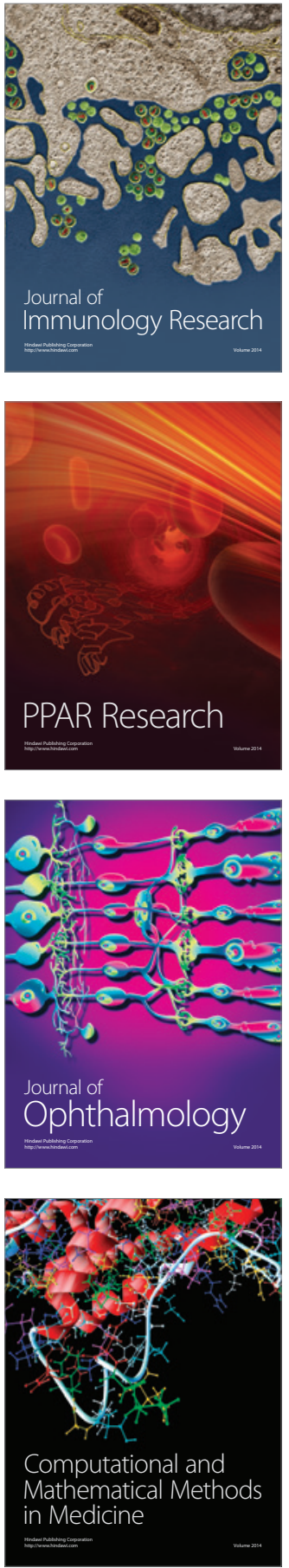

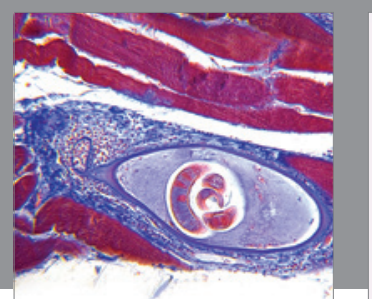

Gastroenterology Research and Practice

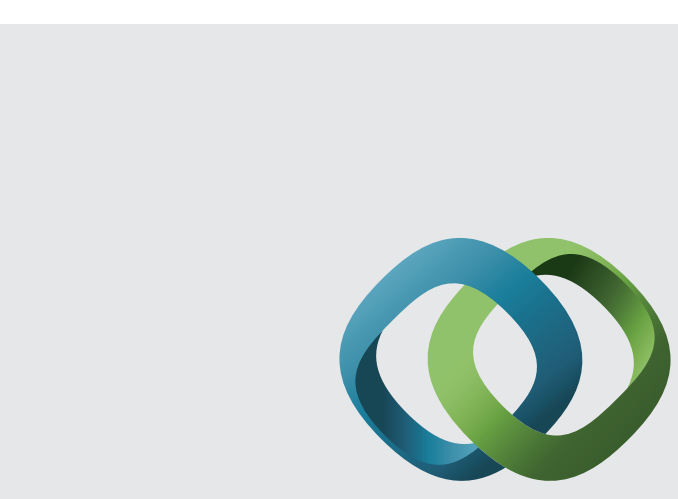

\section{Hindawi}

Submit your manuscripts at

http://www.hindawi.com
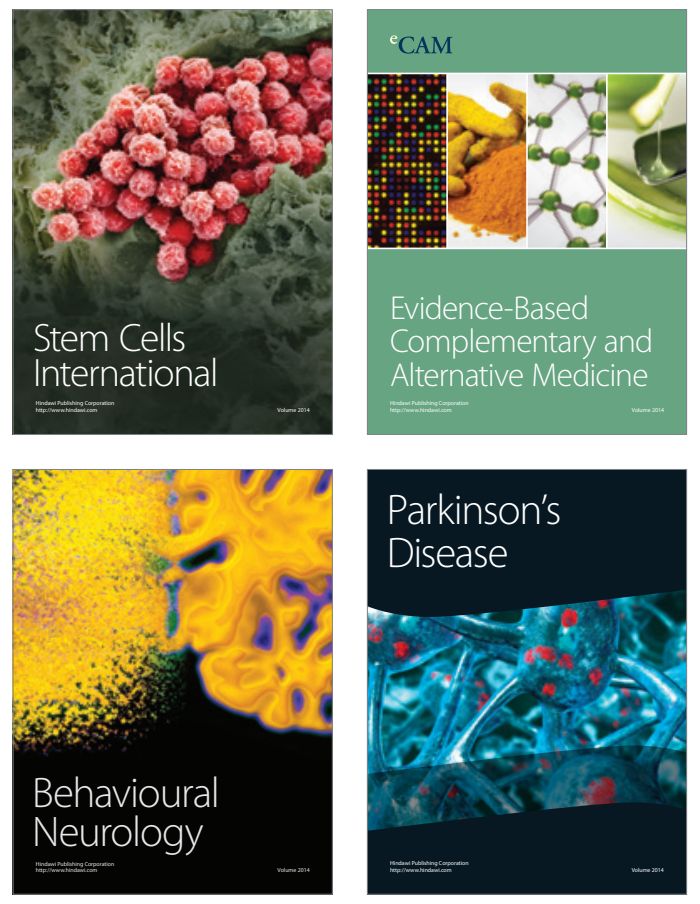
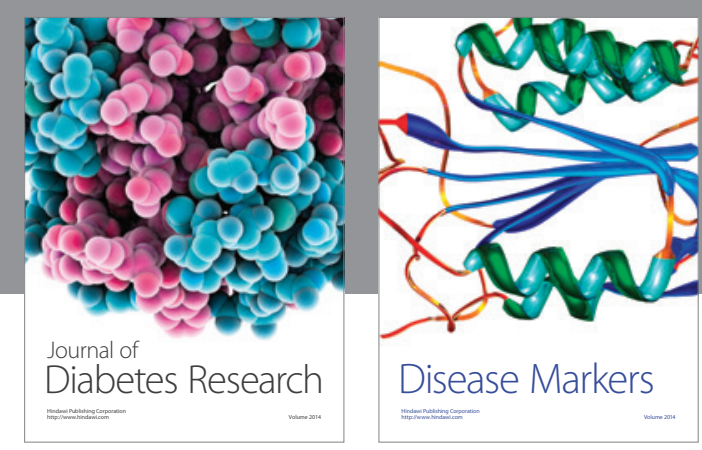

Disease Markers
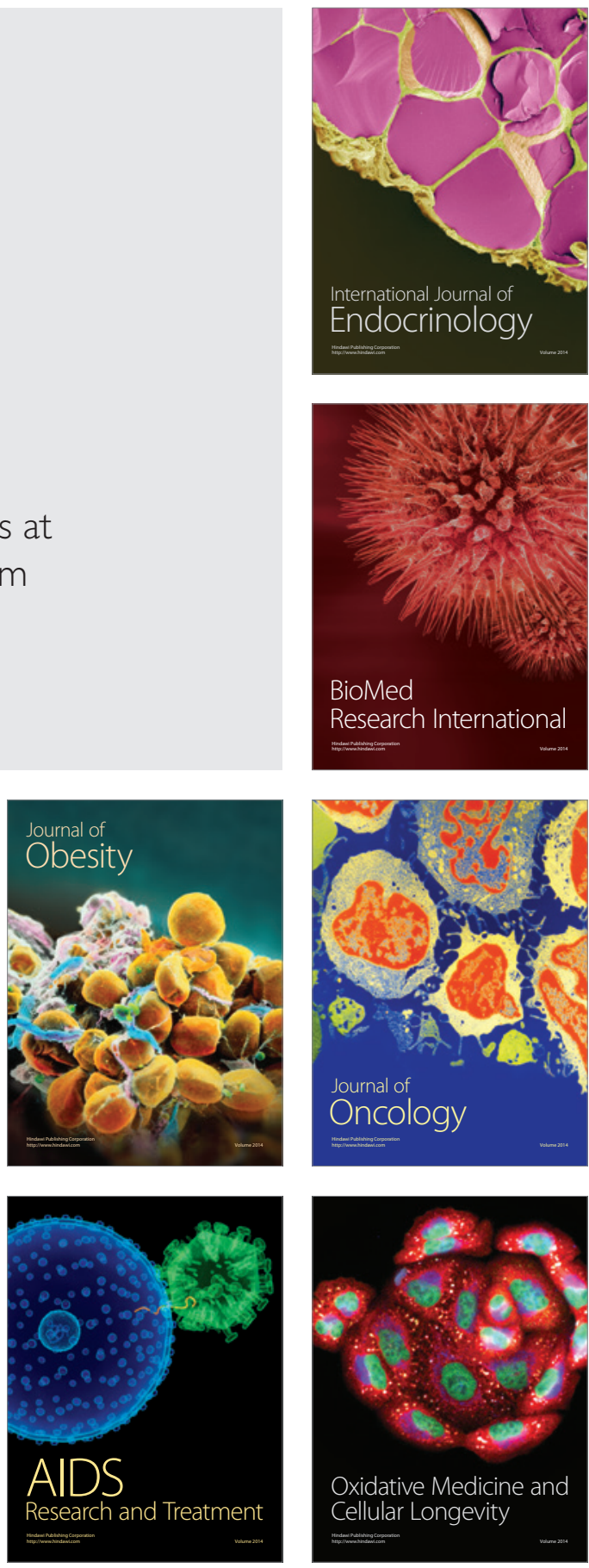